\title{
Application of Handheld Scanner to Investigate Diameter at Breast Height and Tree Height
}

\author{
Joon Kyu Park ${ }^{1}$, Dae Yong Um ${ }^{2}$ \\ ${ }^{1}$ Department of Civil Engineering, Seoil University, 28, Yongmasan-ro 90-gil, \\ Jungnang-gu, Seoul, Korea, \\ ${ }^{2}$ Department of Civil Engineering, Korea National University of Transportation, 50, \\ Daehak-ro, Chungju-si, Chungbuk, Korea, \\ 1jkpark@seoil.ac.kr,2,dyum@ut.ac.kr
}

\begin{abstract}
The Diameter at Breast Height $(D B H)$ is the basic data among the essential items of forest management. Existing DBH survey have generally been made manually. But this way can cause personal and has a disadvantage that it is difficult to measure when the shape of the tree is irregular. In recent years 3D laser scanning method has been introduced in the field of forestry. In this study, DBH and tree height were measured using a 3D laser scanner. Data on the forests studied were obtained using hand-held scanners and DBH and tree height were measured. Additionally obtained data on felled trees was also performed software measurements, and compared the measurements with calipers. The DBH measurement showed a deviation of less than $4 \mathrm{~cm}$ compared to the caliper measurement. This result indicates that the DBH can be measured using a hand-held scanner. And measuring a $1.2 \mathrm{~m}$ high diameter is difficult in the field, so using a handheld scanner will improve work efficiency. The tree height was within 10 $\mathrm{cm}$ of the reference. Although the reference was measured using the total station for accurate measurement, the error of the clinometer used in the field is likely to occur, and the tree height measurement using the hand held scanner may improve the accuracy of the measurement. Further research and measurement automation in the future, the scanner-based method that can be used to reduce noise will be used to measure the diameter of a log tree.
\end{abstract}

Keywords: DBH, Forest, Handheld Scanner, Point Cloud, Measurement

\section{Introduction}

In Korea, it is conducting a survey of national forest resources to obtain basic data on forest statistics and assess the health of forest ecosystems. Forestry in typically using the tools to measure the activities, including size, scope of the tree[1][2][3]. This information has many derivative effects on actual measurers and related tasks or tasks for information users[4][5][6]. Therefore, accurate results are important because they are the basis for research and development related to forests. $\mathrm{DBH}$ is the diameter of a tree measured at 1.2 meters in height[7][8]. DBH is a item for forest research and the most basic data among the essential items of forest management, forest inventory and carbon cycle modeling. Existing DBH measurements were typically performed manually using calipers.

Article history:

Received (May 23, 2019), Review Result (July 6, 2019), Accepted (October 19, 2019) 
However, this method can cause errors in different people, and it is difficult to measure trees if they are irregular in shape. Tree height, along with $\mathrm{DBH}$, is one of the most important factors affecting the volume of trees, so accurate and accurate measurements are required[9][10][11]. In fact, tree height is measured indirectly from clinically to the neck end in $10 \mathrm{~cm}$ increments, and this method also has a high probability of error. 3D laser scanning method was introduced in forestry field with emerging technology[12]. In this study, forestry research using 3D laser scanners was conducted for innovation and application of emerging technologies, and DBH and tree height were measured using 3D laser scanners. Geometric information obtained from 3D laser scanners is actively utilized for reverse engineering and quality inspection of various industries and is expected to be used as a new technology for tree management among mobile technology trends in forestry. Data from the forests studied were obtained using hand-held scanners and measured DBH and tree height.

\section{Data Acquisition and processing}

In this study, experimental forests located in Suwon were selected as research sites. A wellmanaged forest, easy access and a bugle tree that can measure $\mathrm{DBH}$ and tree height were selected for research. GEOSLAM Horizon was used to collect data from the research area. Horizon is a portable 3D laser scanner with an IMU (Inertial Measurement Unit). This equipment gets data as it walks around, so it can acquire data quickly. In addition, automatically registered point clouds can be created using SLAM (simultaneous localization and mapping) technology. Figure 1 shows the shape of the GEOSLAM Horizon.

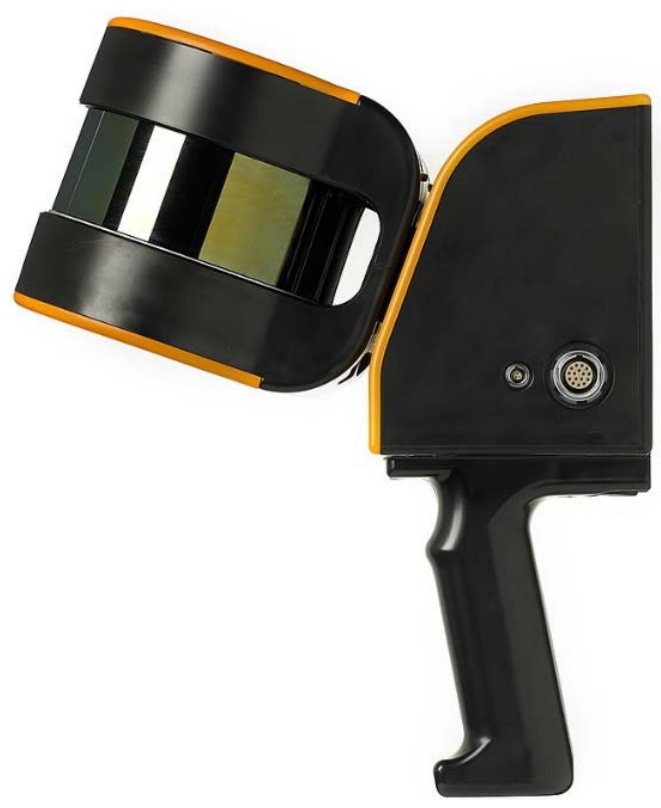

Figure. 1 GEOSLAM Horizon[13]

Data acquired using GEOSLAM Horizon is three-dimensional data in the form of a point cloud. Continuous acquired data is automatically registered using SLAM (simultaneous localization and mapping) technology. Data collection was made for forest areas and deciduous trees. Figure 2 shows the data acquired using GEOSLAM Horizon. 


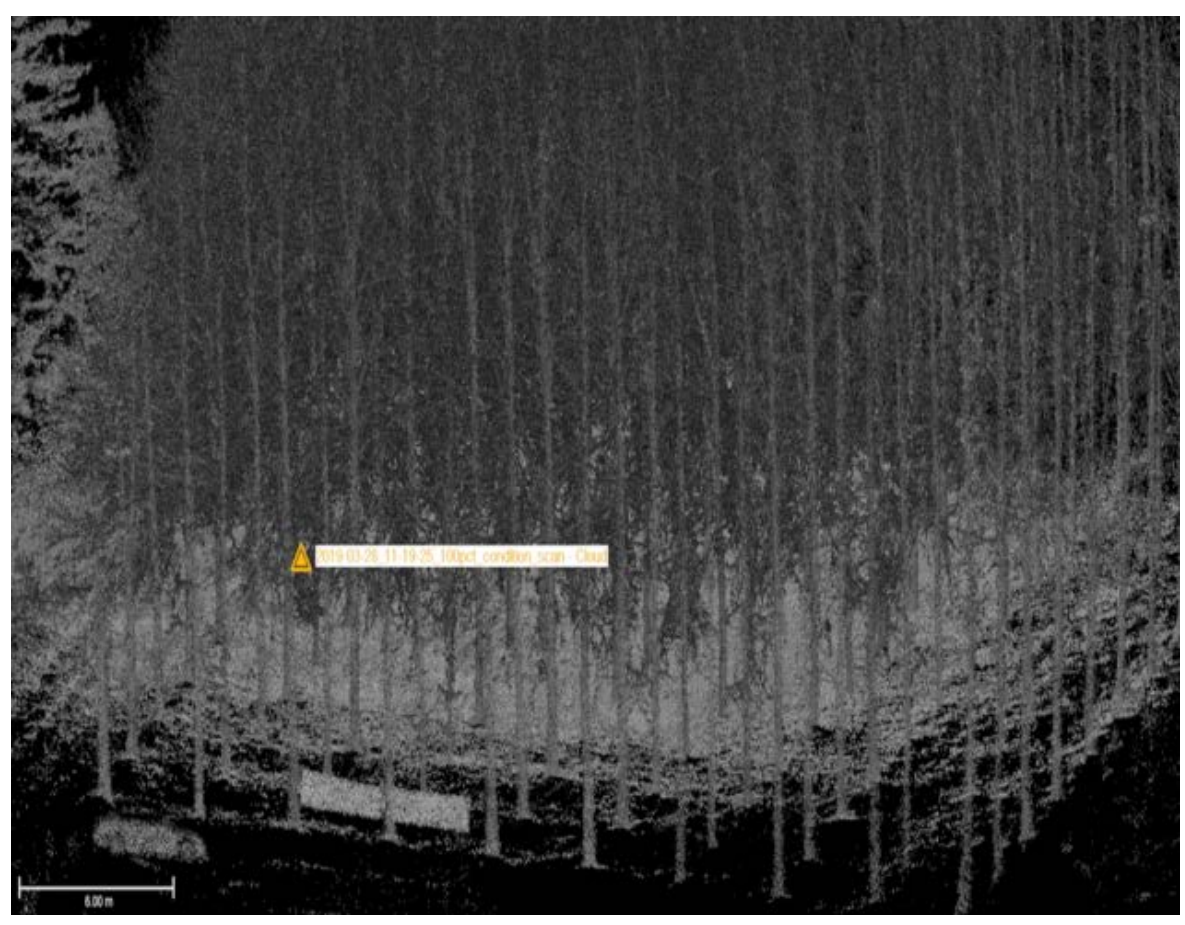

Figure. 2 GEOSLAM Horizon data

The LAS point cloud was created through data processing. The LAS format is typical point cloud data. It can be handled by many general-purpose software programs.

\section{Usability assessment}

In this study, DBH and tree height were measured using point cloud data generated by processing to assess the effectiveness of forest area measurement using hand-held scanners. DBH and tree height were measured using the Simple Real Works software that could edit the point cloud. The land and trees were separated for this purpose. Figure 3 shows the result of separating the trees from the land.

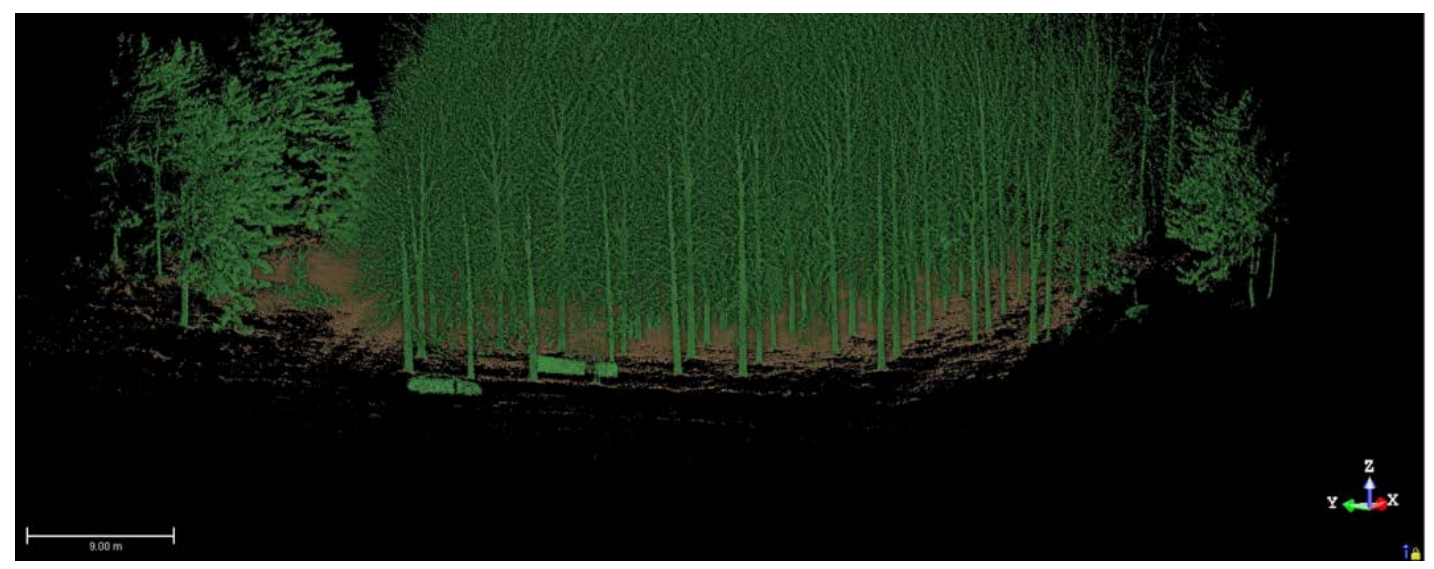

Figure. 3 Separating the tree from the ground 
The measured values of DBH and tree height were compared with calipers and total station values, respectively. Fig. 4 shows the comparison of DBH and tree height.

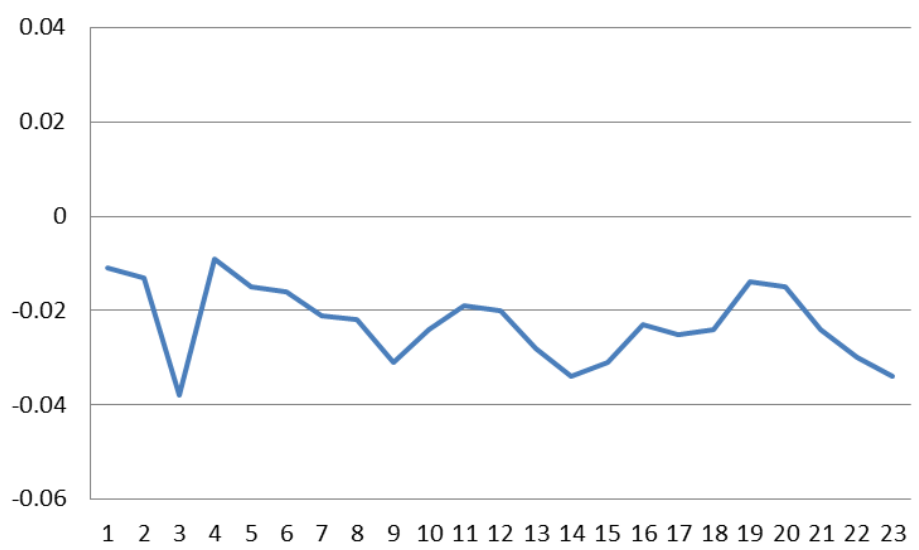

(a) Deviation of DBH

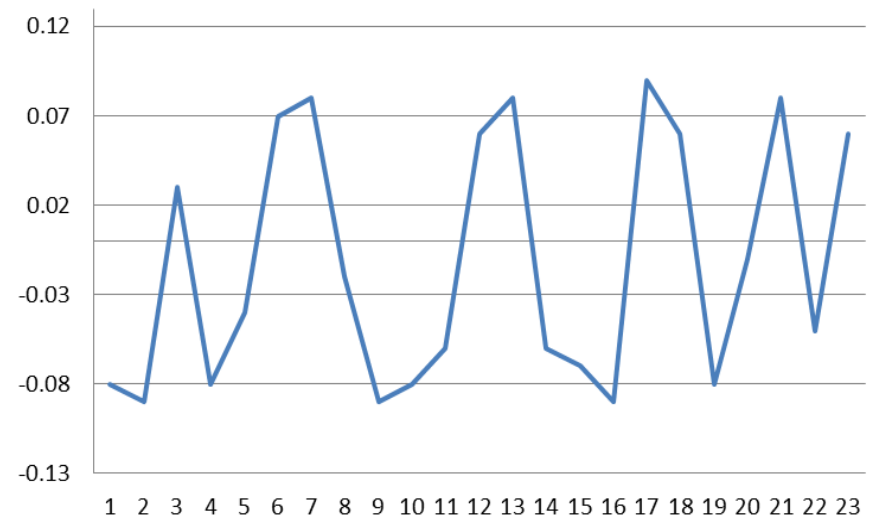

(b) Deviation of tree height

Figure. 4 Comparison of DBH and tree height

As shown in Table 1, DBH measurements showed deviations less than $4 \mathrm{~cm}$ compared to caliper measurements. Research sites are experimental forests, so accurate DBH measurements can be made using calipers. This result indicates that the DBH can be measured using a handheld scanner. This method can reduce errors in measuring calipers of irregularly shaped trees in real forests. In addition, because measuring a 1.2 meter-high diameter is difficult in the field, the use of hand-held scanners can increase work efficiency. The height of the tree was within 10 centimeters of the standard value. For accurate measurements, the baseline was measured using the total station, but there is a possibility of errors in the clinical system used at the site, and tree height measurements using hand held scanners can improve measurement accuracy.

\section{Conclusion}

In this study, forestry research using 3D laser scanners was conducted for innovation and application of emerging technologies, and DBH and tree height were measured using 3D laser scanners. The forest data were obtained using a portable scanner and measured DBH and tree height. Measurements were compared with calipers. The results of this study are as follows. 
1. The DBH measurement showed a deviation of less than $4 \mathrm{~cm}$ compared to the caliper measurement. This result indicates that the DBH can be measured using a portable scanner.

2. The height of the tree was within 10 centimeters of the reference value. For accurate measurements, the baseline was measured using the total station, but there is a possibility of errors in the clinical system used at the site, and tree height measurements using hand held scanners can improve measurement accuracy.

3. Measurements of DBH and tree height using portable scanners will not only increase accuracy, but also improve work efficiency compared to conventional calipers and clinically. The existing method measures and records the $\mathrm{DBH}$ and tree height respectively, but the scanner method is effective because the results can be calculated with one measurement and one office job using software.

\section{Acknowledgements}

This research was supported by Basic Science Research Program through the National Research Foundation of Korea(NRF) funded by the Ministry of Science and ICT(No. NRF2018R1C1B6004021)

\section{References}

[1] H.C. Yun and J.S. Lee, “Accuracy Evaluation and Analysis of SLAM for the Advancement of Forest Investigation”, Journal of the Korea Academia-Industrial cooperation Society, Vol.19, No.12, pp.734-739. (2018) DOI: 10.5762/KAIS.2018.19.12.734

[2] S.H. Ju, S.H. Yoon, S.Y. Park, and J. Heo, "Simulation based Target Geometry Determination Method for Extrinsic Calibration of Multiple 2D Laser Scanning System”, Journal of the Korean Society of Surveying, Geodesy, Photogrammetry and Cartography, Vol.36, No.6, pp.443-449. (2018) DOI: 10.7848/ksgpc.2018.36.6.443

[3] J.H. Oh, Y.J. Jang, and C.N. Lee, “Accuracy Analysis of Low-cost UAV Photogrammetry for Corridor Mapping”, Journal of the Korean Society of Surveying, Geodesy, Photogrammetry and Cartography, Vol.36, No.6, pp.565-572. (2018) DOI: 10.7848/ksgpc.2018.36.6.565

[4] J.K Park and K.Y. Jung, "Investigation and Analysis of Forest Geospatial Information Using Drone” Journal of the Korea Academia-Industrial cooperation Society, vol.19, no.2, pp. 602-607. (2018) DOI: 10.5762/KAIS.2018.19.2.602

[5] H.S. Moon and W.S. Lee, "Development and Verification of A Module for Positioning Buried Persons in Collapsed Area”, Journal of the Korea Academia-Industrial cooperation Society, Vol.17, No.12, pp. 427-436. (2016) DOI: 10.5762/KAIS.2016.17.12.427

[6] K.W. Lee and J.K. Park, "Economic Evaluation of Unmanned Aerial Vehicle for Forest Pest Monitoring”, Journal of the Korea Academia-Industrial cooperation Society, Vol.20, No.1, pp.440-446. (2019)

[7] J.W. Lim, G.E. Park, N.H. Moon, G.H. Moon, and M.Y. Shin, “Analysing the Relationship Between Tree-Ring Growth of Pinus densiflora and Climatic Factors Based on National Forest Inventory Data”, Journal of the Korean Society of Forest Science, Vol.106, No.2, pp.249-257. (2017) DOI: 10.14578/jkfs.2017.106.2.249

[8] J.K. Byun, J.K. Shin, J.E. Yun, H.G. Kim, S.H. Oh, and D.K. Kim, "The Flora of Vascular Plants and Vegetation Type in Mt. Cheonhwang Protected Area for Forest Genetic Resource Conservation, South Korea”, Journal of Agriculture \& Life Science, Vol.51, No.1, pp.77-103. (2017) DOI: 10.11110/kjpt.2013.43.2.146

[9] J. P. Seo, K. D. Kim, and C. S. Woo, “Analysis on Displacement Characteristics of Slow-Moving Landslide on a slope near road Using the Topographic Map and Airborne LiDAR”, Journal of the Korea Academia-Industrial cooperation Society, Vol.20, No.5, pp.27-35. (2019) DOI: 10.5762/KAIS.2019.20.5.27

[10] K. W. Lee and J. K. Park, “Application of Terrestrial LiDAR for Displacement Detecting on Risk Slope”, Journal of the Korea Academia-Industrial cooperation Society, Vol.20, No.1, pp.323-328. (2019) 
[11] S. Y. Park, D. S. Chang, J. H. Jung, Y. J. Kim, and Y. S. Kim, "Prediction of Slope Failure Using Control Chart Method”, Journal of the Korean Geosynthetics Society, Vol.17, No.2, pp.9-18. (2018)

[12] T. D. Acharya, I. T. Yang, and D. H. Lee, "Comparative Analysis of Digital Elevation Models between AW3D30, SRTM30 and Airborne LiDAR: A Case of Chuncheon, South Korea”, Journal of the Korean Society of Surveying, Geodesy, Photogrammetry, and Cartography, Vol.36, No.1, pp.17-24. (2018)

[13] GeoSLAM, (2019), https://geoslam.com/

\section{Authors}

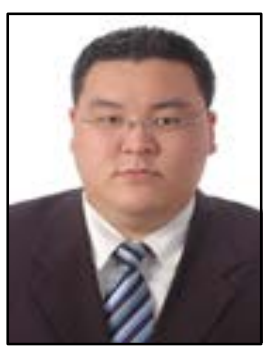

\section{Joon Kyu Park}

He is currently a Professor in Dept. of Civil Engineering at Seoil University. He received his B.S., M.S. and Ph.D. degree in Civil Engineering from Chungnam National University, Korea in 2001, 2003, 2008, respectively. His research interests are in the areas of GPS, GeoSpatial Information Engineering. (jkpark@seoil.ac.kr)

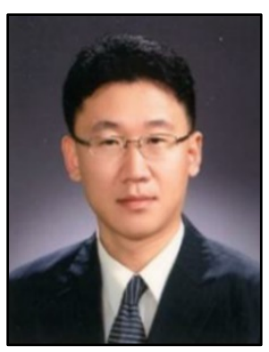

\section{Dae Yong Um}

He is currently a Professor in Dept. of Civil Engineering at Korea National University of Transportation. He received his B.S., M.S. and Ph.D. degree in Civil Engineering from Chungnam National University, Korea in 1997, 1999, 2004, respectively. His research interests are in the areas of Geo-Spatial Information Engineering. (dyum@ut.ac.kr) 